\title{
A modified error-oriented weight positioning model based on DV-Hop
}

\author{
Penghong Wang ${ }^{1,2}$, Xingjuan Cai ${ }^{1}$, and Liping $\mathrm{Xie}^{1 *}$ \\ ${ }^{1}$ School of Computer Science and Technology, Taiyuan University of Science and Technology \\ Taiyuan, 030024 China \\ [e-mail: phwang@hit.edu.cn, xingjuancai@163.com, lipingxie1978@163.com] \\ ${ }^{2}$ Research Center of Intelligent Interface and Human Computer Interaction, Department of Computer Science \\ and Technology, Harbin Institute of Technology \\ Harbin 150006, China \\ [e-mail: phwang@hit.edu.cn] \\ ${ }^{*}$ Corresponding author: Liping Xie
}

Received August 3, 2021; revised Septebmer 28, 2021; accepted October 19, 2021; published February 28, 2022

\begin{abstract}
The distance vector-hop (DV-Hop) is one of the emblematic algorithms that use node connectivity for locating, which often accompanies by a large positioning error. To reduce positioning error, the bio-inspired algorithm and weight optimization model are introduced to address positioning. Most scholars argue that the weight value decreases as the hop counts increases. However, this point of view ignores the intrinsic relationship between the error and weight. To address this issue, this paper constructs the relationship model between error and hop counts based on actual communication characteristics of sensor nodes in wireless sensor network. Additionally, we prove that the error converges to 1/6CR when the hop count increase and tendency to infinity. Finally, this paper presents a modified error-oriented weight positioning model, and implements it with genetic algorithm. The experimental results demonstrate excellent robustness and error removal.
\end{abstract}

Keywords: DV-Hop, modified error-oriented weight model, genetic algorithm, convergence analyzes. 


\section{Introduction}

Internet of things and big data technologies have made great contributions to promoting social development, such as security detection [1, 2], autonomous vehicles [3], risk assessment [4], resources or flow shop scheduling [5-7], and so on [8-11]. Among them, location information plays an important role in biological and industrial production safety [12]. For instance, in the battle against the novel coronavirus disease [13, 14], big data and IoT is used to monitor and predict the spread whereabouts of potential coronavirus cases. And it has played unparalleled roles such the monitoring the flow of suspected people, the distribution of medical personnel, identity authentication. It is worth emphasizing that location information is one of the most indispensable pieces of information during this fight against the epidemic.

Presently, the methods of obtaining location information mainly contain satellite [15] and wireless communication positioning [16]. When under special circumstances such as coal mines and virgin forests, the satellite positioning method loses its positioning advantages and even fails. In this condition, location acquisition mainly depends on wireless communication positioning, including range-free and range-based positioning. Because of economic cost constraints, the range-free positioning method has received more attention. And DV-Hop [17] is a representative algorithm that depends on the location of base station nodes and connectivity between these nodes. The positioning process is as follows:

$\mathbf{1}^{\text {st }}$ step: beacon nodes (BNs) flood the packs to the sensor network, other nodes (including the unknown (UNs) and beacon nodes) record the hop information and BNs' location, then retransmission packs to network. When the connectivity detection is completed, each UN retains only the minimum hop counts between it and $\mathrm{BN}$.

$2^{\text {nd }}$ step: calculated the per hop distance ( Perhop_dis $s_{i}$ ) according to the location and the minimum hop counts between the $B N_{i}$ and $B N_{j}$.

$$
\text { Perhop_dis } s_{i}=\frac{\sum_{i \neq j}\left\|B N_{i}-B N_{j}\right\|}{\sum_{i \neq j} h o p_{i, j}}
$$

where, $B N_{i}$ and $B N_{j}$ refer to the locations of the beacon nodes $\left(B N_{i}\right.$ and $\left.B N_{j}\right), h o p_{i, j}$ refer to the minimum hop count between $B N_{i}$ and $B N_{j}$.

Then, the estimated distance ( $d i_{i, k}$ ) between $B N_{i}$ and $U N_{k}$ is calculated as follows:

$$
\operatorname{dis}_{i, k}=\text { Perhop_dis } s_{i} \cdot \text { hop }_{i, k}
$$

$3^{\text {rd }}$ step: location calculated. In this process, the location of $U N_{k}$ can be obtained by geometric solution or intelligent optimization algorithm. Here, we study the second solution. According to the estimated distance ( $d i s_{i, k}$ ), the location calculation model of $U N_{k}$ is as follows:

$$
f_{i, k}=\sum_{i=1}^{n}|| B N_{i}-U N_{k}\left|-d i s_{i, k}\right|
$$

where $\mathrm{n}$ denotes the number of the $B N_{i}$.

To improve the positioning accuracy, scholars introduced weights to constrain the node $U N_{k}$. The weight calculation model is proposed by Li [18], which is expressed as follows: 


$$
\theta_{i, k}=\frac{1}{h o p_{i, k}}
$$

And the location calculation can express as follows:

$$
f_{i, k}^{*}=\sum_{i=1}^{n} \theta_{i, k}|| B N_{i}-U N_{k}\left|-d i s_{i, k}\right|
$$

This model believes that the greater the hop count, the smallest the weight. Nevertheless, this model ignores the intrinsic relationship between the error and weight, which resulting in a lack of analysis of the model's error convergence. To address this situation, this study analyzes the communication principles of sensor nodes, constructs an error model, and performs convergence analysis. According to the error convergence analysis, a modified error-oriented weight (MEOW) positioning model is proposed and we solve this model with genetic algorithm (GA) [19, 20]. In this paper, the main contributions are as follows: 1) The defects of the current weight model are revealed. 2) A modified error-oriented weight model is constructed; the accuracy of distance estimation has been greatly improved. 3) Extensive simulation experiments are executed to evaluate the performance of the weight model and the effectiveness of the weight model is verified by comparing the classical and the latest algorithms with the equivalent evaluation.

The organization of this study is as follows. Section II introduces the current research status of the weight positioning model and its limitations; Section III proposes a modified error-oriented weight positioning model and analyzes the model convergence. Section IV solves this MEOW positioning model with GA (named MEOWGA-DVHop), and simulates and analyzes it. Finally, the conclusions are drawn in section V.

\section{Related work}

\subsection{Current research status}

For this weight model, scholars have adopted different improvement strategies, which contain deterministic [21-24] and non-deterministic [25-27] strategy improvement. In this paper, we focus on non-deterministic strategies based on optimization algorithms, because the optimization algorithms [28-31] have been proven to have excellent results in various fields [32-35]. Mehrabi [36] used different evolutionary algorithms to optimize the positioning model during the position solution process. Wang [37] revealed the error distribution characteristics of unknown nodes and proposed a multi-objective positioning model based on Gaussian disturbance. Shi [38] introduced a path matching strategy in the model to find the shortest path between beacon nodes, and solved it with PSO. Sharma [39] presented the concept of co-planarity to reduce location errors caused by the anchor nodes which are coplanar, and used the GA solved the weight model. Similarly, Cai [40] presented the fast triangle flip bat algorithm with curve and rank transformation strategy to optimize DV-Hop positioning model. For the problem of uneven distribution of nodes due to the battery life of the nodes, Kanwar [41] presented to localize the newly deployed nodes and use GA to solve the model. Similarity, Prashar [42] presented the error correction metric strategy and PSO to improve DV-Hop localization precision. Shi [43] enhanced the localization accuracy depends on two strategies, which are an N-gram model and a weighed Levenberg-Marquardt method. Han [44] introduced the individual learning strategy of PSO into the DE algorithm to improve the global search ability for unknown nodes. Cai [45] constructed a weight model based on error calculation, but the conditions for the establishment of the model are ideal and the model 
still has deviations in practical applications.

However, what is noteworthy is that during the process of improving the DV-Hop algorithm, scholars have paid more attention to the improvement of the algorithm search strategy, while ignoring the improvement of the weight model. One problem caused by this phenomenon is that the rationality of the weight model as in Eq. (4) has not been verified, and the theoretical basis of the model has not been studied. To address this issue, this paper explored the following research, including the construction of the weight model and calculation of theoretical error.

\subsection{Limitation analysis}

A traditional view is that when the number of hops is $n$, the maximum distance (which is named up bound, UB) that a node can detect is $n \cdot C R$ ( $C R$ denotes communication radius), such as Fig. 1. Fig. 1 is a schematic diagram of the detectable range when hop count is 3. Where $T N_{1}$ and $T N_{2}$ denote the transmit nodes; the dotted circle indicates the communication coverage of the corresponding transmit nodes $\left(T N_{i}\right)$; line segment $(l)$ represents the distance between $B N$ and $U N$. From the Fig. 1, the distance between $B N$ and $U N$ is $3 C R$. Theoretically, only two transmit nodes are needed for $B N$ and $U N$ communication, which requires two transmit nodes to be in the same location as $T N_{1}$ and $T N_{2}$. However, according to the relevant knowledge of probability theory, we conduct the following analysis.

Assumption: In the detection area, event $A$ indicates that a node a coincides with node $T N_{1}$, whose probability is expressed as $P(A)$; and event $B$ indicates that a node a coincides with node $T N_{2}$, whose probability is expressed as $P(B)$. And events $A$ and $B$ are independent of each other. Therefore, we have $P(A)=P(B)=0$ (Because in the plane, the value of probability that two points coincide is 0 .). Thus, the probability of simultaneous occurrence of event $A$ and $B$ can be expressed as $P(A B)=P(A) P(B)=0$. That is, in the actual communication process, the ideal communication situation shown in Fig. 1 does not exist.

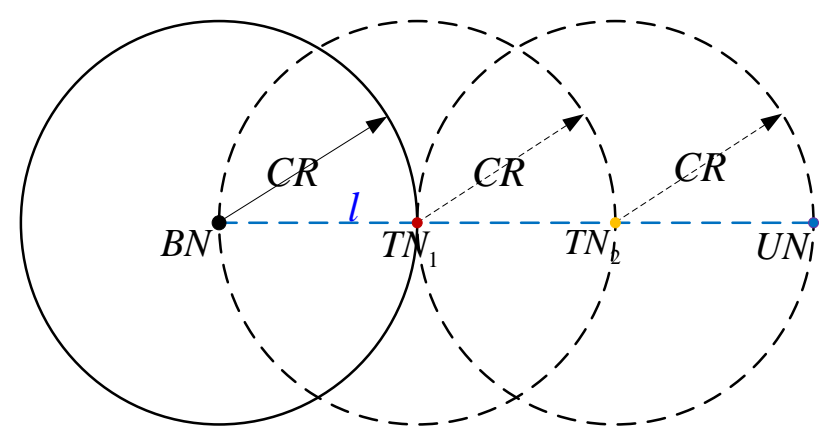

Fig. 1. Schematic diagram of detectable range when the number of hops is 3 .

Therefore, when the hop count is $n$, the maximum detectable distance of node is smaller than $n \cdot C R$. And it means the traditional model has defects during the detection distance analysis process, so that the constructed weight model cannot reflect the actual communication characteristics of the node. To tackle this defect, and to construct a weight model more in line with the characteristics of actual communication, this work conducts the following research. 


\section{Modified estimated distance calculation model}

According section 2.2, we can know that when the value of hop count is $\mathrm{n}$ in the actual communication process, the maximum detection distance (up bound) is less than $n \cdot C R$. To address this issue, this section models the estimated distance and error of the nodes based on the actual communication characteristics. For convenience, the following variables are introduced: $a_{n}, b_{n}$ and $\varphi_{n}$. Where, $a_{n}$ denotes the average up bound (AUB), $b_{n}$ indicates the theoretical estimation distance, and $\varphi_{n}$ denotes the error when the value of hop is $n$. (Remark: Because the upper boundary is less than $n \cdot C R$ when the value of hop is $n$, the concept of AUB is introduced when constructing the model, which is considered to be the maximum upper boundary obtained by probability.).

\subsection{Model analysis when the hop count is 1}

When the hop count is 1 , the distribution of nodes is shown in Fig. 2. Where, $r_{k}$ indicates the distance between the $U N_{k}$ and $B N$, and $d r$ denotes the variable ( $\Delta r$ ) between the node $U N_{k}$ and $U N_{k-1}$. From Fig. 2, we can know that when the hop count is 1, the communication between nodes does not require retransmission; therefore, the value of UB is CR. Correspondingly, when the number of nodes $k$ tends to $\infty$, average per hop distance of beacon node can be expressed as the average of the distance sum of nodes $U N_{k}$ to $B N$, that is mean $\left(\sum_{1}^{\infty} r_{k}\right)$. And the calculation is expressed as follows:

$$
\text { Per_dis }=\frac{\int_{0}^{C R} 2 \pi r^{2} d r}{\int_{0}^{C R} 2 \pi r d r}=\frac{2}{3} C R
$$

where, $P e r_{\text {_dis }}$ indicates the theoretical average distance per hop.

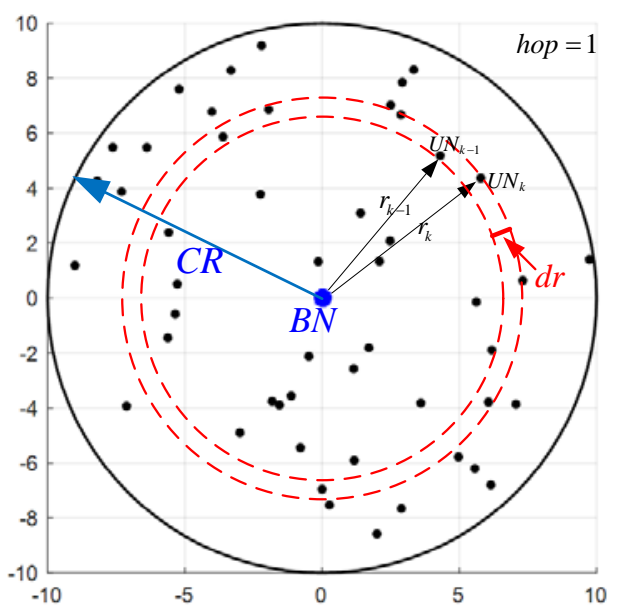

Fig. 2. Analysis of theoretical estimated distance.

According to the value of equation (1) and (6), we can know that the value of Perhop_dis is distributed on both sides of $\frac{2}{3} C R$. Therefore, we regard Per_dis as the theoretical estimation distance $\left(b_{1}\right)$ when the hop count 1 . The corresponding error distribution characteristics can be expressed as Fig. 3. 
Thus, we have $a_{1}=C R, b_{1}=\frac{2}{3} C R$, and the error $\varphi_{1}$ is calculated as follows:

$$
\varphi_{1}=\frac{\int_{a_{0}}^{b_{1}} 2 \pi r\left(b_{1}-r\right) d r+\int_{b_{1}}^{a_{1}} 2 \pi r\left(r-b_{1}\right) d r}{\int_{a_{0}}^{a_{1}} 2 \pi r d r}=\frac{16}{81} C R
$$

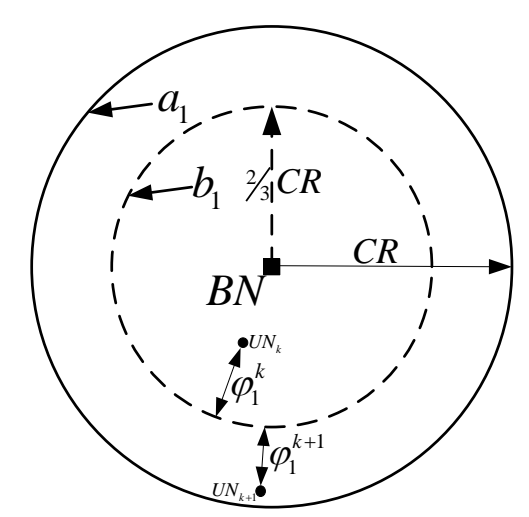

Fig. 3. Analysis of theoretical estimated distance.

\subsection{Estimated distance analysis when the hop count is $\mathbf{n}$}

When the hop count is 2 , a relay node is needed for mutual communication between nodes. Due to cost constraints, the actual deployment number of sensor nodes is limited, which will cause the loss of communication distance as shown in Fig. 4. From the Fig. 4, the distance between the node $T N$ and the radius is $\gamma$ (where, $\gamma$ indicates the communication distance loss), which bring the communication distance loss is $\gamma$ when forwarding by this node. And it reveals when the value of hop is $n$, the actual communication distance is less than $n \cdot C R$.

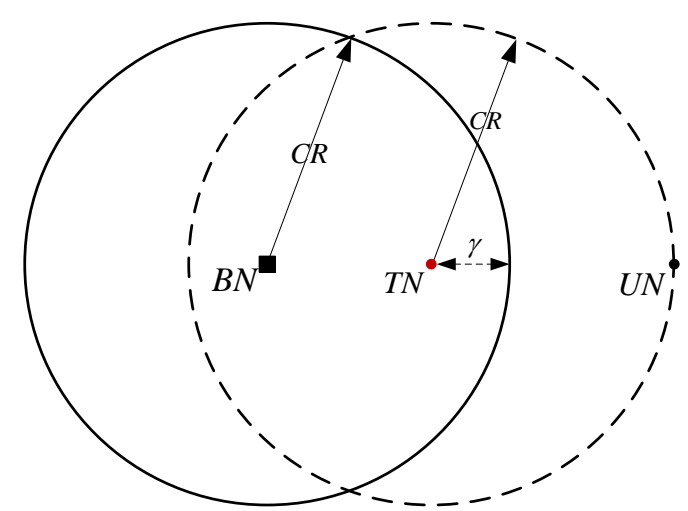

Fig. 4. Communication distance loss analysis when the number of hops is 2.

Against this phenomenon, we propose the distance estimated model shown in Fig. 5. Given: $a_{1}=C R, b_{1}=\frac{2}{2} C R$; therefore, the $a_{2}$ is calculated as $b_{1}+C R$. It introduces the concept of the average upper boundary (AUB), and the distance loss generated by each node is statistically processed. Therefore, the maximum detection distance is approximated as $b_{1}+C R$ when the hop count is 2 . 
Accordingly, the $b_{2}$ is calculated as follows (it denotes the average distance between node $B N$ and other sensor nodes in interval $\left.\left[a_{1}, a_{2}\right]\right)$.

$$
b_{2}=\frac{\int_{a_{1}}^{a_{2}} 2 \pi r^{2} d r}{\int_{a_{1}}^{a_{2}} 2 \pi r d r}=\frac{\int_{C R}^{\frac{5}{3} C R} 2 \pi r^{2} d r}{\int_{C R}^{\frac{5}{c} C R} 2 \pi r d r}=\frac{14}{9} C R
$$

Further, when the value of hop is 3 (n), the distance estimated model is shown in Fig. 6. Similarly, $a_{3}$ can be calculated as $b_{2}+C R$, and the $a_{n}$ can be expressed as:

$$
a_{n}=b_{n-1}+C R
$$

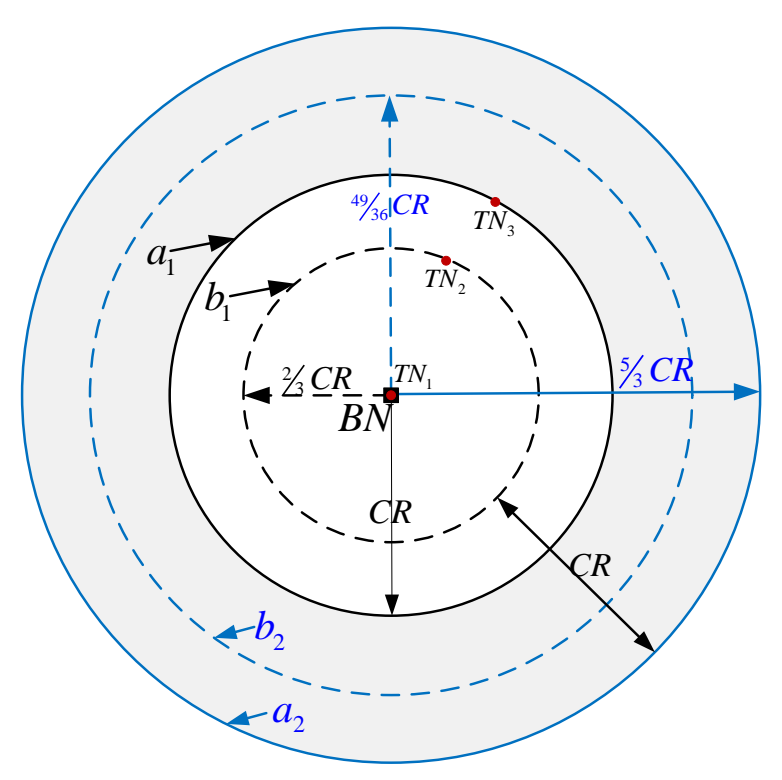

Fig. 5. Distance estimated model when the number of hops is 2 .

Accordingly, the $b_{n}$ can be expressed as:

$$
b_{n}=\frac{\int_{a_{n-1}}^{a_{n}} 2 \pi r^{2} d r}{\int_{a_{n-1}}^{a_{n}} 2 \pi r d r}=\frac{2}{3}\left(a_{n}+a_{n-1}\right)-\frac{2}{3} \frac{a_{n} a_{n-1}}{a_{n}+a_{n-1}}
$$




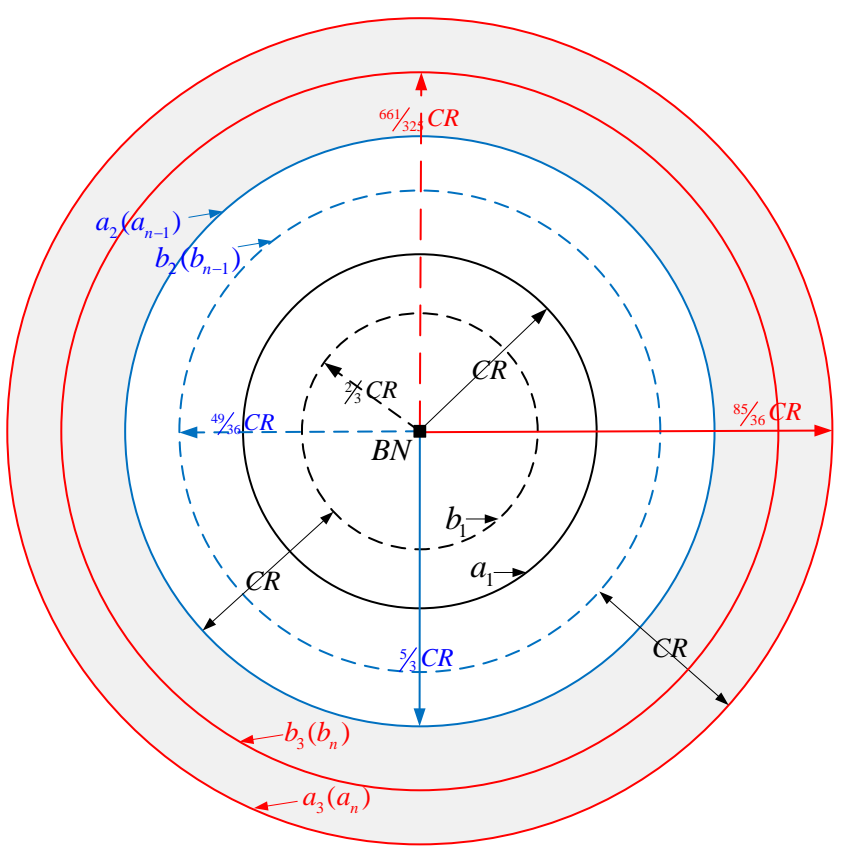

Fig. 6. Estimated distance model when the number of hops is 3(n).

\subsection{Error analysis with the hop increases}

According to the Eq. (7), we can know when the value of hop is n; the error can be denoted as a sectional integral on interval $\left[a_{n-1}, a_{n}\right]$, where the dividing line is $b_{n}$. And the error analysis is shown in Fig. 7.

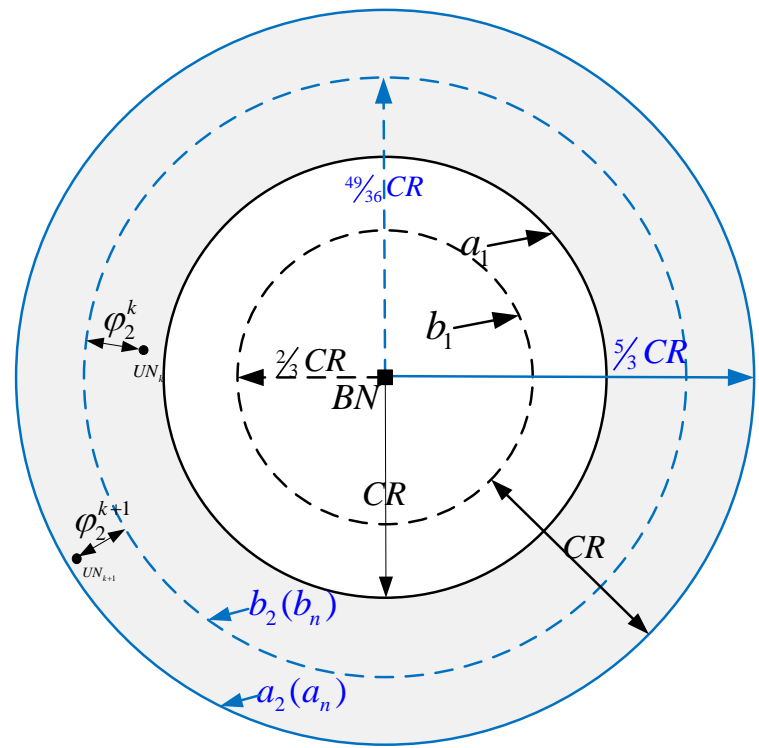

Fig. 7. Error analyze when the value of hop is n.

Correspondingly, the error calculation can be expressed as: 


$$
\begin{aligned}
\varphi_{n} & =\frac{\int_{a_{n-1}}^{b_{n}} 2 \pi r\left(b_{n}-r\right) d r+\int_{b_{n}}^{a_{n}} 2 \pi r\left(r-b_{n}\right) d r}{\int_{a_{n-1}}^{a_{n}} 2 \pi r d r} \\
& =\frac{\frac{2}{3} b_{n}^{3}-b_{n}\left(a_{n-1}^{2}+a_{n}^{2}\right)+\frac{2}{3}\left(a_{n-1}^{3}+a_{n}^{3}\right)}{a_{n}^{2}-a_{n-1}^{2}}
\end{aligned}
$$

Then, the error convergence is analyzed. According to the Eq. (9) and (10), we can know that the $a_{n}$ and $b_{n}$ are recursive expressions. Thus, we explore the relationship between $a_{n}$ and $b_{n}$, and found when the hop count tendency to $\infty$, the convergence of $a_{n}-b_{n}$ can be expressed as:

$$
\lim _{n \rightarrow \infty}\left(a_{n}-b_{n}\right)=\lim _{n \rightarrow \infty} b_{n-1}+C R-\left(\frac{2}{3}\left(a_{n}+a_{n-1}\right)-\frac{2}{3} \frac{a_{n} a_{n-1}}{a_{n}+a_{n-1}}\right)=\frac{1}{3} C R
$$

Combined with Eq. (9), we have:

$$
\left.\begin{array}{l}
\lim _{n \rightarrow \infty}\left(a_{n}-b_{n}\right)=\frac{1}{3} C R \\
a_{n}=b_{n-1}+C R
\end{array}\right\} \Rightarrow\left\{\begin{array}{l}
\lim _{n \rightarrow \infty}\left(b_{n}-b_{n-1}\right)=\frac{2}{3} C R \\
\lim _{n \rightarrow \infty}\left(b_{n}-a_{n-1}\right)=\frac{1}{3} C R
\end{array}\right.
$$

Therefore, when the $n$ tends to $\infty$, the value of $b_{n}-b_{n-1}$ converges to $\frac{2}{3} C R$, and the value of $b_{n}-a_{n-1}$ converges to $1 / 3 C R$. Accordingly, the convergence of error $\varphi_{n}$ can be expressed as:

$$
\lim _{n \rightarrow \infty} \varphi_{n}=\lim _{n \rightarrow \infty} \frac{\frac{2}{3} b_{n}^{3}-b_{n}\left(a_{n-1}^{2}+a_{n}^{2}\right)+\frac{2}{3}\left(a_{n-1}^{3}+a_{n}^{3}\right)}{a_{n}^{2}-a_{n-1}^{2}}=\frac{1}{6} C R
$$

Therefore, in actual communication conditions, when the hop count increase and tendency to $\infty$, the error $\varphi_{n}$ converges to $1 / 6 C R$.

\section{Modified error-oriented weight model}

\subsection{Construction of error model}

The weight is an important embodiment of the influence of beacon nodes on unknown nodes. According to the error analysis of the nth hop, this study improves the traditional weight model and proposes a modified error-oriented weight (MEOW) model. The Fig. 8 reflects the details of the error changes with the number of hops. Where, Fig. 8 (a) the reflects the change in error when the maximum hop count is 20, and (b) reflects the change trend of the error when the maximum hop count is 100. Apparently, as the number of hops increases, the error shows a downward trend of fluctuations, and it reveals the tradition weight model (which is Eq. (4)) is inaccurate. 


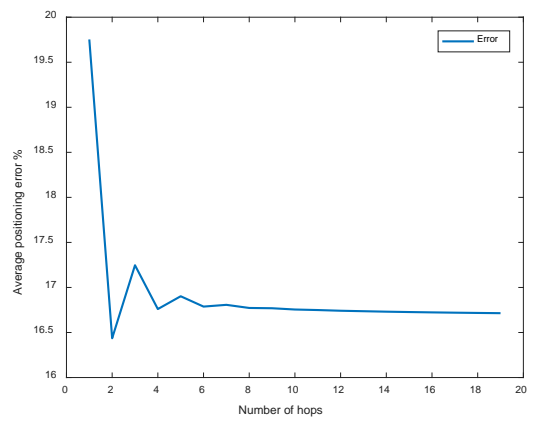

(a)

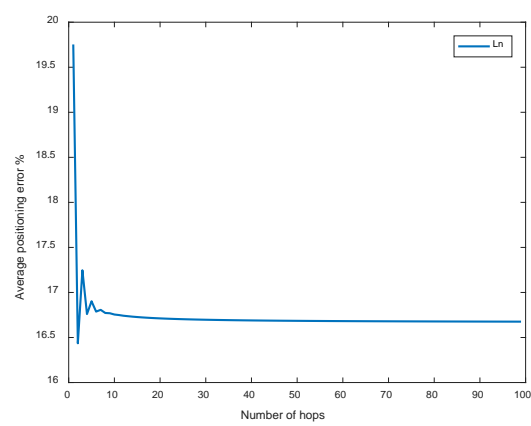

(b)

Fig. 8. The graph of error. (a) the maximum value is 20 , (b) the maximum value is 100 .

Considering the idea that the larger the weight, the smaller the error, we constructed the following weight model:

$$
\omega_{i, k}=\frac{1}{\varphi_{i, k}}, i=1,2, \ldots, n
$$

where, $\omega_{i, k}$ represents the weight of node $B N_{i}$ to node $U N_{k}$, and $\varphi_{i, k}$ denotes the theoretical error between the node $B N_{i}$ to node $U N_{k}$.

And the fitness evaluation model is:

$$
f_{i, k}=\sum_{i=1}^{n} \omega_{i, k}|| B N_{i}-U N_{k}\left|-d_{i, k}\right|
$$
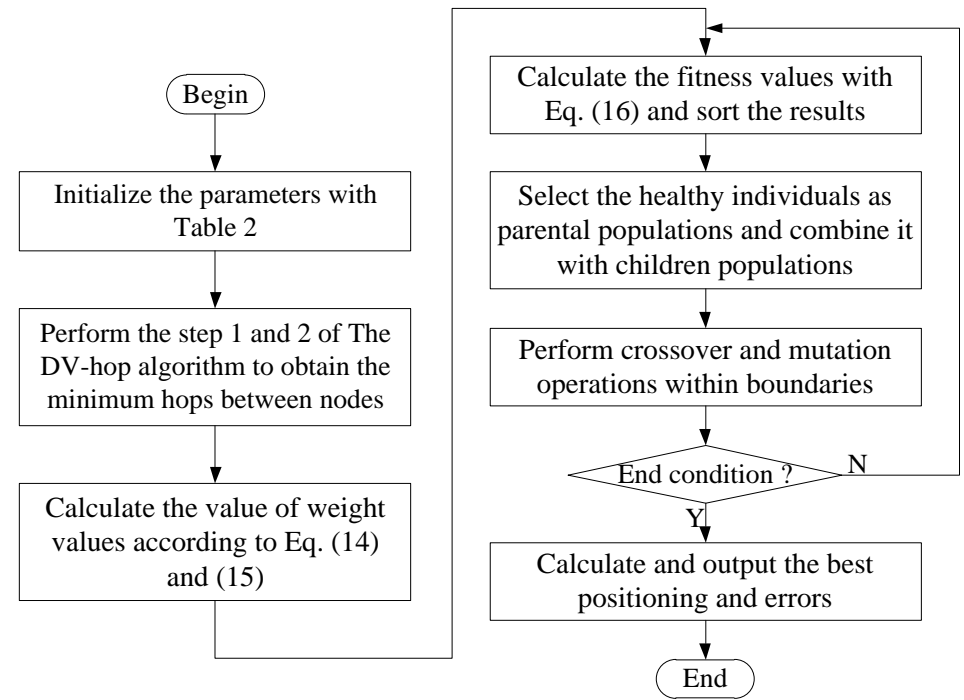

Fig. 9. Flow chart of MEOWGA-DVHop.

\subsection{Solution of weight model}

This section uses GA to solve the weight model, and the process is shown in Fig. 9. The algorithm framework includes two parts: DV-Hop algorithm and genetic algorithm. The left shows the first two stages of DV-Hop algorithm, and the right shows the algorithm flow of genetic algorithm. 
During this execution of the algorithm, to ensure the convergence of the algorithm, the crossover and mutation operations are performed with a certain probability. And the calculation of the error follows Eq. (18).

\section{Simulation tests}

\subsection{Model construction rationality analysis}

To verify the rationality of the construction of the weight model, this section makes a statistical analysis of the estimated distance of the model, the simulation parameters are shown in Table 1 (Remark: The nodes follow the Uniform distribution in the detection area, and the nodes are regenerated each time they are tested independently.).

Table 1. Simulation parameters

\begin{tabular}{|c|c|}
\hline Parameters & Value \\
\hline Detection area & $200 \mathrm{~m} \times 200 \mathrm{~m}$ \\
\hline Nodes (N) & 300 \\
\hline BNs (n) & 20 \\
\hline R (m) & 25 \\
\hline Independently runtime & 100 \\
\hline
\end{tabular}

According to the step 1 and 2 of DV-Hop algorithms, record the minimum hops required for nodes to communicate with each other, and mark the actual distance between nodes when the hop count is 3 . The results are shown in Fig. 10. In ideal conditions, when the number of hops is 3 , the maximum detection distance is $75 \mathrm{~m}$. However, in the statistical results, the maximum detection distance is only $71 \mathrm{~m}$, which is less than the theoretical value of $75 \mathrm{~m}$. The resulting fitting function is expressed as follows:

$$
f(x)=0.56 \cdot \mathrm{e}^{-\left(\frac{x-53.2}{11.1}\right)^{2}}
$$

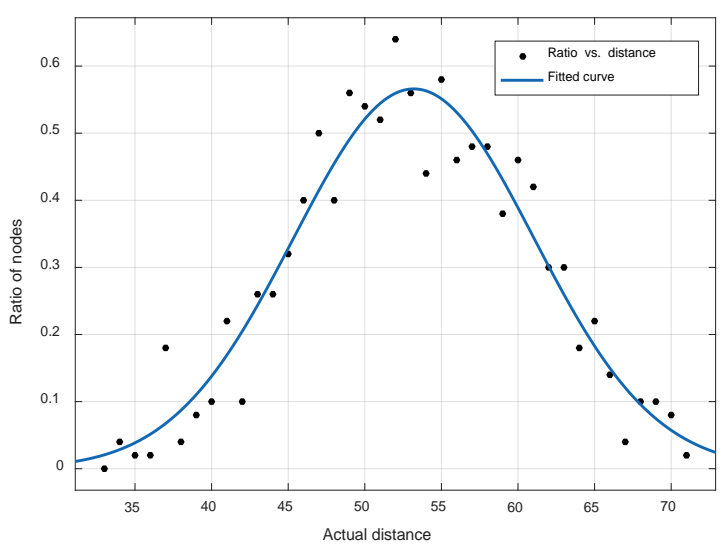

Fig. 10. Actual distance statistics of nodes when the hop count is 3 .

According to the Eq. (17), we know that the standard deviation interval is [42.1m, $64.3 \mathrm{~m}]$. 
In contrast, the interval of detection distance in the ideal (traditional view) case is [50 m, $75 \mathrm{~m}]$ (where, the detection distance interval in ideal conditions is $[(n-1) \cdot C R, n \cdot C R]$ ); the interval of detection distance in this work is $[34.03 \mathrm{~m}, 59.03 \mathrm{~m}]$. Apparently, the interval similarity in this work is $76.26 \%$, the interval similarity in ideal conditions is $64.41 \%$. It illustrates that the weight model (distance estimation model) constructed in this paper is obviously superior to the traditional weight model.

\subsection{Positioning error analysis}

This section mainly compares and analyzes the positioning errors of nodes to test the performance of the proposed model. The program is shown in Fig. 9, and the algorithm parameters are shown in Table 2. The test results are compared with DV-Hop, OCS-DVHop [26], TWPSO-DVHop [38] and TWGA-DVHop [39].

Table 2. Simulation parameters

\begin{tabular}{|c|c|c|c|}
\hline Parameters & Value & Parameters & Value \\
\hline Network type & C-shaped, O-shaped, X-shaped & Population & 20 \\
\hline Area & $100 \mathrm{~m} \times 100 \mathrm{~m}$ & Variable dimension $(\mathrm{v})$ & $2 \mathrm{M}$ \\
\hline Nodes & $100(50-100)$ & Cross probability & 0.9 \\
\hline BNs & $20(5-30)$ & Mutation probability & $1 / \mathrm{v}$ \\
\hline CR $(\mathrm{m})$ & $25(15-40)$ & Maximum iterations & 500 \\
\hline-- & -- & Independently runtime & 30 \\
\hline
\end{tabular}

The calculation of node error can be expressed as follows:

$$
A P E=\frac{1}{M \cdot R} \sum_{j=1}^{M}\left\|U N_{j}^{*}-U N_{j}\right\| \cdot 100 \%
$$

Where, APE denotes the average positioning error, $M$ indicates the number of $U N s, U N_{j}^{*}$ indicates the estimated location, and $U N_{j}$ denotes the actual location.

Additionally, to test the robustness of this model, the test set used in this paper is the same as in literature 30, which contains three different network topologies, such as Fig. 11.

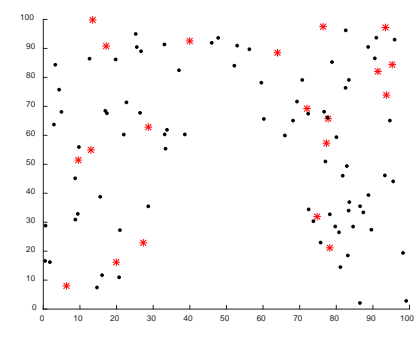

(a)

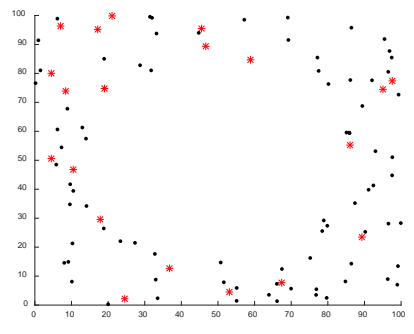

(b)

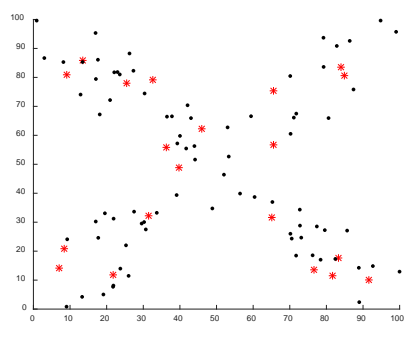

(c)

Fig. 11. Different complex networks topologies. (a): Type-C, (b): Type-O, (c): Type-X.

\subsection{Different CR test}

The Table 3 and Fig. 12 reveal the positioning error within the different CR. Apparently, compare with the standard DV-Hop and other traditional weight positioning algorithms, 
MEOWGA-DVHop shows outstanding positioning performance. When the value of CR is 15, the positioning errors respectively drop $101.5 \%, 70.22 \%$ and $37.8 \%$. Especially, both TWGA-DVHop and MEOWGA-DVHop use the GA solve model, the difference is that TWGA-DVHop solves the traditional weight model, and MEOWGA-DVHop solves the modified error-oriented model. From the results, we can see that the error of MEOWGA-DVHop is much smaller than TWGA-DVHop, which reflects the advantages of the model.

Table 3. The results under different CR

\begin{tabular}{|c|c|cccccc|}
\hline \multicolumn{1}{|c|}{ CR } & $\mathbf{1 5}$ & $\mathbf{2 0}$ & $\mathbf{2 5}$ & $\mathbf{3 0}$ & $\mathbf{3 5}$ & $\mathbf{4 0}$ \\
\hline \multirow{5}{*}{ Type-C } & DV-Hop & 172.33 & 112.53 & 63.73 & 49.78 & 44.81 & 41.62 \\
& OCS-DVHop & 81.98 & 58.59 & 37.35 & 30.46 & 32.09 & 29.36 \\
& TWPSO-DVHop & 93.76 & 63.01 & 36.30 & 31.20 & 29.51 & 27.56 \\
& TWGA-DVHop & $\mathbf{5 0 . 9 9}$ & $\mathbf{3 9 . 2 8}$ & $\mathbf{3 1 . 3 2}$ & 31.17 & 30.96 & 31.64 \\
& MEOWGA-DVHop & 70.83 & 52.59 & 34.09 & $\mathbf{2 9 . 5 7}$ & $\mathbf{2 8 . 8 6}$ & $\mathbf{2 7 . 3 0}$ \\
\hline \multirow{5}{*}{ Type-O } & DV-Hop & 117.88 & 56.50 & 44.77 & 39.39 & 29.24 & 31.28 \\
& OCS-DVHop & 49.32 & 31.05 & 23.77 & 26.86 & 20.85 & 21.98 \\
& TWPSO-DVHop & 50.58 & 27.28 & 24.33 & 24.74 & 18.06 & 20.58 \\
& TWGA-DVHop & $\mathbf{4 4 . 1 4}$ & 31.77 & 28.15 & 30.39 & 25.70 & 28.37 \\
& MEOWGA-DVHop & 47.66 & $\mathbf{2 5 . 7 4}$ & $\mathbf{2 2 . 4 4}$ & $\mathbf{2 3 . 0 7}$ & $\mathbf{1 7 . 5 5}$ & $\mathbf{1 9 . 9 8}$ \\
\hline \multirow{5}{*}{ Type-X } & DV-Hop & 80.18 & 54.22 & 43.49 & 39.39 & 37.15 & 36.29 \\
& OCS-DVHop & 45.68 & 33.60 & 35.84 & 32.43 & 30.41 & $\mathbf{2 6 . 6 0}$ \\
& TWPSO-DVHop & 45.06 & 32.52 & 34.23 & 34.42 & 28.43 & 27.41 \\
& TWGA-DVHop & $\mathbf{4 1 . 5 7}$ & 36.93 & 38.60 & 37.58 & 35.50 & 34.59 \\
& MEOWGA-DVHop & $\mathbf{4 2 . 3 8}$ & $\mathbf{3 1 . 4 6}$ & $\mathbf{2 9 . 5 9}$ & $\mathbf{2 9 . 0 7}$ & $\mathbf{2 5 . 9 0}$ & 26.78 \\
\hline
\end{tabular}

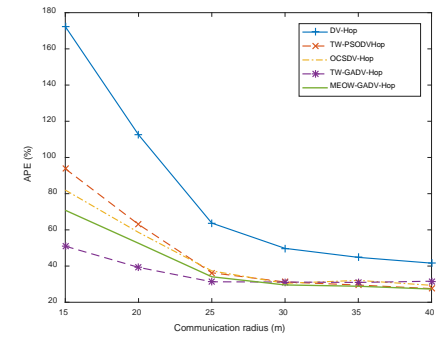

(a)

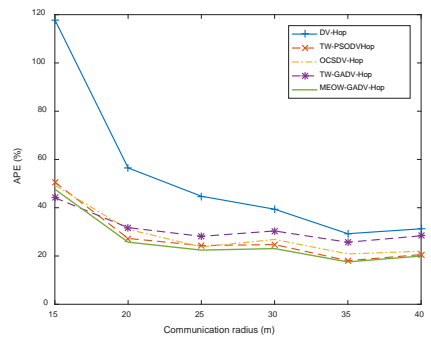

(b)

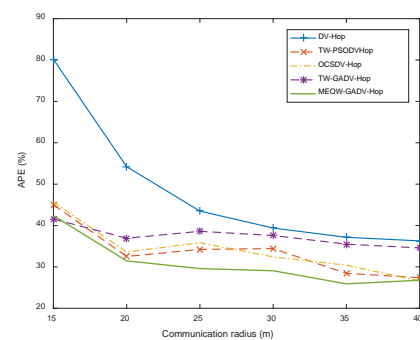

(c)

Fig. 12. The APE within different CR. (a): Type-C, (b): Type-O, (c): Type-X.

Specially, we can see that there are some values of APE greater than 100\% in Fig. 12. Why does this happen? As shown in Fig. 13, it belongs to Type-O networks. And there are two labeled nodes $B N_{i}$ and $U N_{k}$, and the distance between them is $70.4784 \mathrm{~m}$. When the CR is $15 \mathrm{~m}$, the two nodes need four relay nodes (five hops) to communicate with each other in ideal conditions. However, in actual deployment, there may be no deployed nodes between them, like Fig. 13. At this time, we need additional relay nodes to maintain the connectivity of the two nodes. In Fig. 13, we need 15 such relay nodes (16 hops). There is no doubt that this will bring a big error, according to the APE calculation equation (Eq. 18), there is a probability that the APE values are greater than 100. And with the CR increases, the value of APE decreases rapidly. 


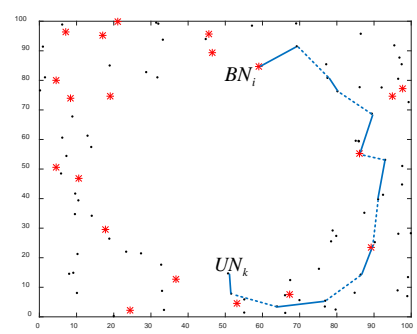

Fig. 13. The information transfer diagram of nodes $B N_{i}$ and $U N_{k}$ communicating with each other.

\subsection{Different CR test}

The Table 4 and Fig. 14 reveal the positioning error within the different nodes. In Type-C network, the error of MEOWGA-DVHop is larger than TWGA-DVHop, but smaller than other comparison algorithms. In Type-O and Type-X networks, the results show that the MEOWGA-DVHop always keeps the minimum localization error.

Table 4. The results under different nodes

\begin{tabular}{|c|c|cccccc|}
\hline \multicolumn{1}{|c|}{} & Nodes & $\mathbf{5 0}$ & $\mathbf{6 0}$ & $\mathbf{7 0}$ & $\mathbf{8 0}$ & $\mathbf{9 0}$ & $\mathbf{1 0 0}$ \\
\hline \multirow{5}{*}{ Type-C } & DV-Hop & 76.27 & 75.39 & 70.34 & 66.42 & 65.12 & 63.73 \\
& OCS-DVHop & 43.98 & 43.07 & 40.63 & 39.64 & 38.68 & 37.35 \\
& TWPSO-DVHop & 41.70 & 45.09 & 39.05 & 38.47 & 37.40 & 36.30 \\
& TWGA-DVHop & $\mathbf{3 2 . 3 5}$ & $\mathbf{3 5 . 1 1}$ & $\mathbf{3 3 . 3 8}$ & $\mathbf{3 3 . 1 5}$ & $\mathbf{3 0 . 6 8}$ & $\mathbf{3 1 . 3 2}$ \\
& MEOWGA-DVHop & 40.51 & 43.32 & 37.70 & 36.90 & 34.95 & 34.09 \\
\hline \multirow{5}{*}{ Type-O } & DV-Hop & 33.92 & 40.59 & 40.82 & 41.80 & 42.46 & 44.77 \\
& OCS-DVHop & 21.63 & 23.48 & 23.12 & 23.31 & 22.84 & 23.77 \\
& TWPSO-DVHop & 20.15 & 24.81 & 22.96 & 23.59 & 23.89 & 24.33 \\
& TWGA-DVHop & 22.38 & 27.36 & 25.40 & 26.20 & 26.50 & 28.15 \\
& MEOWGA-DVHop & $\mathbf{1 9 . 5 8}$ & $\mathbf{2 2 . 8 4}$ & $\mathbf{2 1 . 4 3}$ & $\mathbf{2 1 . 7 1}$ & $\mathbf{2 2 . 4 9}$ & $\mathbf{2 2 . 4 4}$ \\
\hline \multirow{5}{*}{ Type-X } & DEV-Hop & 34.16 & 36.47 & 38.00 & 40.31 & 40.30 & 43.49 \\
& OCS-DVHop & 35.34 & 34.21 & 35.27 & 35.86 & 35.13 & 35.84 \\
& TWPSO-DVHop & 35.24 & 32.78 & 31.65 & 31.76 & 31.76 & 34.23 \\
& TWGA-DVHop & 34.85 & 32.58 & 34.44 & 35.89 & 36.56 & 38.60 \\
& MEOWGA-DVHop & $\mathbf{3 1 . 6 4}$ & $\mathbf{2 9 . 6 0}$ & $\mathbf{2 9 . 0 6}$ & $\mathbf{2 8 . 8 9}$ & $\mathbf{2 8 . 6 4}$ & $\mathbf{2 9 . 5 9}$ \\
\hline \multirow{5}{*}{} & & & & & & &
\end{tabular}

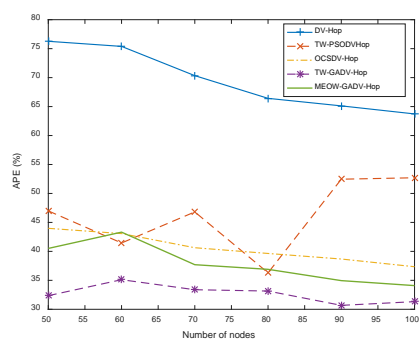

(a)

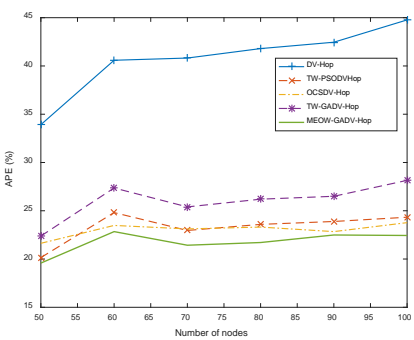

(b)

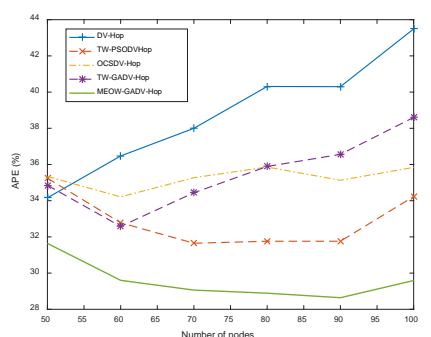

(c)

Fig. 14. The APE within different nodes. (a): Type-C, (b): Type-O, (c): Type-X.

And from the Fig. 14, we can know that MEOWGA-DVHop is not sensitive to node density, and there are two reasons causing this situation: one is the model we built is more accurate than traditional models, such as Fig. 10; and another is GA has a strong global 
convergence ability.

\subsection{Different anchor nodes test}

The Table 5 and Fig. 15 reveal the positioning error within the different number of BNs. In Type-C network, when the number of BNs is smaller than 20, the positional accuracy of MEOWGA-DVHop is superior to the TWGA-DVHop, and inferior to it when the number of BNs is larger than 20. And in other networks, the error of MEOWGA-DVHop is significantly superior to other algorithms, contains DV-hop, OCS-DVHop, TWPSO-DVHop and TWGA-DVHop algorithms.

Table 5. The results under different BNs

\begin{tabular}{|c|c|rccccc|}
\hline \multicolumn{1}{|c|}{ BNs } & $\mathbf{5}$ & $\mathbf{1 0}$ & $\mathbf{1 5}$ & $\mathbf{2 0}$ & $\mathbf{2 5}$ & $\mathbf{3 0}$ \\
\hline \multirow{5}{*}{ Type-C } & DV-Hop & 88.45 & 67.42 & 69.45 & 63.73 & 64.88 & 69.80 \\
& OCS-DVHop & 102.36 & 49.62 & 41.73 & 37.35 & 51.77 & 52.90 \\
& TWPSO-DVHop & $\mathbf{4 6 . 9 9}$ & 41.45 & 46.81 & 36.30 & 52.46 & 52.71 \\
& TWGA-DVHop & 116.76 & 43.28 & 37.54 & $\mathbf{3 1 . 3 2}$ & $\mathbf{3 3 . 2 1}$ & $\mathbf{3 1 . 3 0}$ \\
& MEOWGA-DVHop & 73.28 & $\mathbf{3 7 . 3 6}$ & $\mathbf{4 0 . 3 0}$ & 34.09 & 38.73 & 38.11 \\
\hline \multirow{5}{*}{ Type-O } & DV-Hop & 98.08 & 79.95 & 38.47 & 44.77 & 38.28 & 40.49 \\
& OCS-DVHop & 45.15 & 36.60 & 33.17 & 23.77 & 34.99 & 35.72 \\
& TWPSO-DVHop & $\mathbf{3 0 . 7 5}$ & 33.09 & 28.90 & 24.33 & 32.71 & 31.72 \\
& TWGA-DVHop & 76.28 & 51.68 & 30.23 & 28.15 & 26.26 & 25.00 \\
& MEOWGA-DVHop & 46.92 & $\mathbf{3 2 . 4 4}$ & $\mathbf{2 3 . 0 6}$ & $\mathbf{2 2 . 4 4}$ & $\mathbf{2 2 . 9 9}$ & $\mathbf{2 1 . 3 7}$ \\
\hline \multirow{5}{*}{ Type-X $X$} & DV-Hop & 58.46 & 59.14 & 47.89 & 43.49 & 46.66 & 48.57 \\
& OCS-DVHop & 48.83 & 39.74 & 46.47 & 35.84 & 45.32 & 45.87 \\
& TWPSO-DVHop & 41.99 & 39.80 & 41.92 & 34.23 & 47.04 & 46.36 \\
& TWGA-DVHop & 60.86 & 42.36 & 38.83 & 38.60 & 36.91 & 33.12 \\
& MEOWGA-DVHop & $\mathbf{4 4 . 2 3}$ & $\mathbf{3 2 . 6 8}$ & $\mathbf{3 0 . 3 3}$ & $\mathbf{2 9 . 5 9}$ & $\mathbf{2 9 . 7 8}$ & $\mathbf{2 5 . 5 1}$ \\
\hline
\end{tabular}

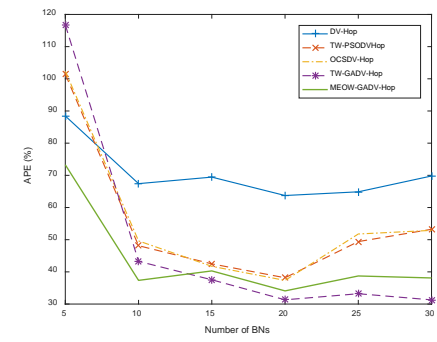

(a)

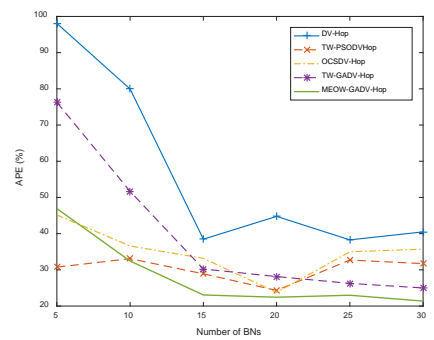

(b)

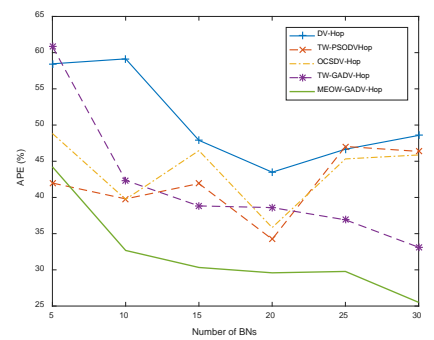

(c)

Fig. 15. The APE within different BNs. (a): Type-C, (b): Type-O, (c): Type-X.

\subsection{Time complexity}

The time complexity of the comparison algorithm is shown in Table 6. Where, the MaxIt indicates the maximum iterations, NP denotes the population size. Because both TWGA-DVHop and MEOWGA-DVHop use GA to solve the model, the time complexity of them is identical. Similarly, the OCS-DVHop and TWPSO-DVHop has identical time complexity. And the time complexity of MEOWGA-DVHop is smaller than OCS-DVHop. 
Table 6. Time complexity

\begin{tabular}{|c|c|}
\hline Algorithms & time complexity \\
\hline OCS-DVHop & $O($ MaxIt $\cdot(\mathrm{N}-\mathrm{n}) \cdot N P \log N P)$ \\
\hline TWPSO-DVHop & $O($ MaxIt $\cdot(\mathrm{N}-\mathrm{n}) \cdot N P \log N P)$ \\
\hline TWGA-DVHop & $O($ MaxIt $\cdot N P \log N P)$ \\
\hline MEOWGA-DVHop & $O($ MaxIt $\cdot N P \log N P)$ \\
\hline
\end{tabular}

\section{Conclusions}

Aiming at the lack of convergence analysis among intelligent positioning algorithms in WSNs, this paper builds an intrinsic relationship model between error and hops based on the characteristics of sensor nodes in the actual communication process among the component systems. This model reveals that the theoretical value of error will converge to a constant $\left(\frac{1}{6} C R\right.$ ), when the hop counts tend to infinity (Remark: There are a certain number of sensor nodes in the detection area, and they are following the Uniform distribution.). Then, we present the modified error-oriented weight (MEOW) positioning model and solve it with GA. Specially, this paper considers the distance loss in the actual communication process, which makes the constructed distance estimation model more accurate. Such as Fig. 10, when the hop value is 3 , the interval similarity between the estimated distance and the actual distance of the traditional weight model is only $65 \%$. The modified model proposed in this paper can be increased to $79 \%$, this makes it more advantageous in the positioning process. And the simulation results confirm that the MEOWGA-DVHop is significantly superior to other algorithms.

In addition, there are some defects in MEOWGA-DVHop proposed in this paper from the test results of the Type-C network. And our next step is to explore the possible causes of this phenomenon and continue to optimize the positioning model. And it will also be included in subsequent research that the deployment in real-world applications for the developed model.

\section{Acknowledgement}

This work is supported by the National Natural Science Foundation of China under Grant No.61806138, Key Research and Development Project of Shanxi Province (High Technology) under Grant No.201903D121119. Special thanks should go to my partner Tian Fan who has put considerable time and effort into their comments on this paper.

\section{References}

[1] Z. X. Zhang, Y. Cao, Z. H. Cui, W. S. Zhang, and J. J. Chen, "A Many-Objective Optimization Based Intelligent Intrusion Detection Algorithm for Enhancing Security of Vehicular Networks in 6G," IEEE Transactions on Vehicular Technology, vol. 70, no. 6, pp. 5234-5243, 2021. Article (CrossRef Link)

[2] Z. H. Cui, Y. R. Zhao, Y. Cao, X. J. Cai, W. S. Zhang, and J. J. Chen, "Malicious Code Detection under 5G HetNets Based on a Multi-Objective RBM Model," IEEE Network, vol. 35, no. 2, pp. 82-87, 2021. Article (CrossRef Link)

[3] L. Wang and B. K. P. Horn, "Time-to-contact control: improving safety and reliability of autonomous vehicles," International Journal of Bio-Inspired Computation, vol. 16, no. 2, pp. 68-78, 2020. Article (CrossRef Link) 
[4] Y. Zeng and F. Yu, "Improvement and simulation of cost risk assessment model for intelligent building engineering," International Journal of Computing Science and Mathematics, vol. 12, no. 2, pp. 177-191, 2020. Article (CrossRef Link)

[5] Cai, X., Geng, S., Wu, D., Cai, J., \& Chen, J., “A Multicloud-Model-Based Many-Objective Intelligent Algorithm for Efficient Task Scheduling in Internet of Things,” IEEE Internet of Things Journal, vol. 8, no. 12, pp. 9645-9653, 2021. Article (CrossRef Link)

[6] X. Zhang, X. T. Li, and M. H. Yin, "An enhanced genetic algorithm for the distributed assembly permutation flowshop scheduling problem," International Journal of Bio-Inspired Computation, vol. 15, no. 2, pp. 113-124, 2020. Article (CrossRef Link)

[7] W. Wang, H. Wang, and C. Li, "Decision preference-based artificial bee colony algorithm for many-objective optimal allocation of water resources," International Journal of Computing Science and Mathematics, vol. 12, no. 4, pp. 364-373, 2020. Article (CrossRef Link)

[8] X. Cai, Y. Cao, Y. Ren, Z. Cui, W. Zhang, "Multi-objective Evolutionary 3D Face Reconstruction Based on Improved Encoder-Decoder Network,” Information Sciences, vol. 581, pp. 233-248, 2021. Article (CrossRef Link)

[9] Cui, Z., Zhao, P., Hu, Z., Cai, X., Zhang, W., \& Chen, J., “An improved matrix factorization based model for many-objective optimization recommendation,” Information Sciences, vol. 579, pp. 1-14, 2021. Article (CrossRef Link)

[10] Cai, X., Hu, Z., \& Chen, J., "A many-objective optimization recommendation algorithm based on knowledge mining,” Information Sciences, vol. 537, pp. 148-161, 2020. Article (CrossRef Link)

[11] Z., Cui, X., Jing, P., Zhao, W., Zhang, \& J. Chen, “A new subspace clustering strategy for AI-based data analysis in IoT system,” IEEE Internet of Things Journal, vol. 8, no. 16, pp. 12540-12549, 2021. Article (CrossRef Link)

[12] J. Machaj, P. Brida, and N. Majer, "Challenges introduced by heterogeneous devices for Wi-Fi-based indoor localization," Concurrency and Computation-Practice \& Experience, vol. 32, no. 13, 2020, Art. no. e5198. Article (CrossRef Link)

[13] F. Zhou et al., "Clinical course and risk factors for mortality of adult inpatients with COVID-19 in Wuhan, China: a retrospective cohort study," Lancet, vol. 395, no. 10229, pp. 1054-1062, 2020. Article (CrossRef Link)

[14] H. S. Shi et al., "Radiological findings from 81 patients with COVID-19 pneumonia in Wuhan, China: a descriptive study," Lancet Infectious Diseases, vol. 20, no. 4, pp. 425-434, 2020. Article (CrossRef Link)

[15] P. Enge, "The Global Positioning System: Signals, measurements, and performance," IJWIN, vol. 1, pp. 83-105, 1994. Article (CrossRef Link)

[16] T. Nebbou, M. Lehsaini, H. Fouchal, and M. Ayaida, "An urban location service for vehicular area networks," Concurrency and Computation-Practice \& Experience, vol. 31, no. 24, 2019, Art. no. e4693. Article (CrossRef Link)

[17] D. Niculescu and B. Nath, "DV based positioning in Ad Hoc networks," Telecommunication Systems, vol. 22, no. 1-4, pp. 267-280, 2003. Article (CrossRef Link)

[18] J. Li, J. Zhang, and X. Liu, "A Weighted DV-Hop Localization Scheme for Wireless Sensor Networks," in Proc. of International Conference on Scalable Computing and Communications; Eighth International Conference on Embedded Computing, Scalcom-Embeddedcom, pp. 269-272, 2009. Article (CrossRef Link)

[19] D. Goldberg, Genetic Algorithm in Search, Optimization, and Machine Learning, Addison-Wesley, Reading, Massachusetts, pp. 2104-2116, 1989. [Online]. Available: https://dl.acm.org/doi/book/10.5555/534133.

[20] Y. Kaniovski, Y. Kaniovskyi, and G. Pflug, "Analysis of credit-rating migrations with genetic algorithms," International Journal of Bio-Inspired Computation, vol. 16, no. 4, pp. 264-274, 2020. Article (CrossRef Link)

[21] J. Li, J. Zhang, and L. Xiande, "A Weighted DV-Hop Localization Scheme for Wireless Sensor Networks," in Proc. of 2009 International Conference on Scalable Computing and Communications; Eighth International Conference on Embedded Computing, pp. 269-272, 2009. Article (CrossRef Link) 
[22] Y. Hu and X. M. Li, "An improvement of DV-Hop localization algorithm for wireless sensor networks," Telecommunication Systems, vol. 53, no. 1, pp. 13-18, 2013. Article (CrossRef Link)

[23] W. Zhao, S. B. Su, and F. Shao, "Improved DV-Hop Algorithm Using Locally Weighted Linear Regression in Anisotropic Wireless Sensor Networks," Wireless Personal Communications, vol. 98, no. 4, pp. 3335-3353, 2018. Article (CrossRef Link)

[24] X. Y. Yan, L. J. Sun, J. Zhou, and A. G. Song, "DV-hop localization algorithm based on optimal weighted least square in irregular areas," Electronics Letters, vol. 54, no. 21, pp. 1243-1244, 2018. Article (CrossRef Link)

[25] Y. Gao, Y. Zhuang, T. Ni, K. Yin, and T. Xue, "An improved genetic algorithm for wireless sensor networks localization," in Proc. of 2010 IEEE Fifth International Conference on Bio-Inspired Computing: Theories and Applications (BIC-TA), pp. 439-443, 2010. Article (CrossRef Link)

[26] Z. H. Cui, B. Sun, G. G. Wang, Y. Xue, and J. J. Chen, "A novel oriented cuckoo search algorithm to improve DV-Hop performance for cyber-physical systems," Journal of Parallel and Distributed Computing, vol. 103, pp. 42-52, 2017. Article (CrossRef Link)

[27] L. Z. Cui, C. Xu, G. H. Li, Z. Ming, Y. H. Feng, and N. Lu, "A high accurate localization algorithm with DV-Hop and differential evolution for wireless sensor network," Applied Soft Computing, vol. 68, pp. 39-52, 2018. Article (CrossRef Link)

[28] W. Chen, J. Ye, R. Wu, G. Liu, and P. Kang, "Firefly algorithm based on intelligent single particle learning," International Journal of Computing Science and Mathematics, vol. 12, no. 4, pp. 309-326, 2020. Article (CrossRef Link)

[29] X. Cai, S. Geng, D. Wu, J. Chen, "Unified integration of many-objective optimization algorithm based on temporary offspring for software defects prediction," Swarm and Evolutionary Computation, vol. 63, pp. 100871, 2021. Article (CrossRef Link)

[30] Cui, Z., Zhang, J., Wu, D., Cai, X., Wang, H., Zhang, W., \& Chen, J., "Hybrid many-objective particle swarm optimization algorithm for green coal production problem," Information Sciences, vol. 518, pp. 256-271, 2020. Article (CrossRef Link)

[31] F. Xue and D. Wu, "NSGA-III algorithm with maximum ranking strategy for many-objective optimisation," International Journal of Bio-Inspired Computation, vol. 15, no. 1, pp. 14-23, 2020. Article (CrossRef Link)

[32] J. Wang, "A hybrid grid-based many-objective optimization algorithm for software defect prediction," International Journal of Computing Science and Mathematics, vol. 12, no. 4, pp. 374-384, 2020. Article (CrossRef Link)

[33] C. H. Xu, A. S. Ding, and S. S. Liao, "A privacy-preserving recommendation method based on multi-objective optimisation for mobile users," International Journal of Bio-Inspired Computation, vol. 16, no. 1, pp. 23-32, 2020. Article (CrossRef Link)

[34] N. Kumar, M. S. Rahman, A. Duary, S. K. Mahato, and A. K. Bhunia, "A new QPSO based hybrid algorithm for bound-constrained optimisation problem and its application in engineering design problems," International Journal of Computing Science and Mathematics, vol. 12, no. 4, pp. 385-412, 2020. Article (CrossRef Link)

[35] S. S. Choong, L. P. Wong, M. Y. H. Low, and C. S. Chong, "A bee colony optimisation algorithm with a sequential-pattern-mining-based pruning strategy for the travelling salesman problem," International Journal of Bio-Inspired Computation, vol. 15, no. 4, pp. 239-253, 2020. Article (CrossRef Link)

[36] M. Mehrabi, H. Taheri, and P. Taghdiri, "An improved DV-Hop localization algorithm based on evolutionary algorithms," Telecommunication Systems, vol. 64, no. 4, pp. 639-647, Apr 2017. Article (CrossRef Link)

[37] P. H. Wang, J. R. Huang, Z. H. Cui, L. P. Xie, and J. J. Chen, "A Gaussian error correction multi-objective positioning model with NSGA-II," Concurrency and Computation-Practice \& Experience, vol. 32, no. 5, p. e5464, 2020. Article (CrossRef Link)

[38] Q. Shi, Q. Xu, and J. Zhang, "An Improved DV-Hop Scheme Based on Path Matching and Particle Swarm Optimization Algorithm," Wireless Personal Communications, vol. 104, no. 4, pp. 1301-1320, 2019. Article (CrossRef Link) 
[39] G. Sharma and A. Kumar, "Improved range-free localization for three-dimensional wireless sensor networks using genetic algorithm," Computers \& Electrical Engineering, vol. 72, pp. 808-827, 2018. Article (CrossRef Link)

[40] X. J. Cai, S. J. Geng, P. H. Wang, L. Wang, and Q. D. Wu, "Fast triangle flip bat algorithm based on curve strategy and rank transformation to improve DV-Hop performance," Ksii Transactions on Internet and Information Systems, vol. 13, no. 12, pp. 5785-5804, 2019. Article (CrossRef Link)

[41] V. Kanwar and A. Kumar, "DV-Hop based localization methods for additionally deployed nodes in wireless sensor network using genetic algorithm," Journal of Ambient Intelligence and Humanized Computing, vol. 11, pp. 5513-5531, 2020. Article (CrossRef Link)

[42] D. Prashar and D. Kumar, "Performance Evaluation of the Optimized Error Correction Based Hop Localization Approach in a Wireless Sensor Network," Wireless Personal Communications, vol. 111, no. 4, pp. 2517-2543, 2020. Article (CrossRef Link)

[43] Q. Q. Shi, Q. Xu, and J. P. Zhang, "Amended DV-hop scheme based on N-gram model and weighed LM algorithm," Electronics Letters, vol. 56, no. 5, pp. 247-250, 2020. Article (CrossRef Link)

[44] D. Z. Han, Y. P. Yu, K. C. Li, and R. F. de Mello, "Enhancing the Sensor Node Localization Algorithm Based on Improved DV-Hop and DE Algorithms in Wireless Sensor Networks," Sensors, vol. 20, no. 2, pp. 343, 2020. Article (CrossRef Link)

[45] X. J. Cai, P. H. Wang, Z. H. Cui, W. S. Zhang, and J. J. Chen, "Weight convergence analysis of DV-hop localization algorithm with GA," Soft Computing, vol. 24, no. 23, pp. 18249-18258, 2020. Article (CrossRef Link)

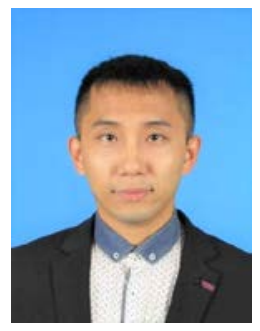

Penghong Wang received the M.S. degrees from Computer Science and Technology, Taiyuan University of Science and Technology, Taiyuan, China, in 2020. He is pursuing his Ph.D. degree in School of Computer Science at Harbin Institute of Technology (HIT), Harbin 150001, China. His main research interests include wireless sensor network, distributed source-channel coding and computer vision.

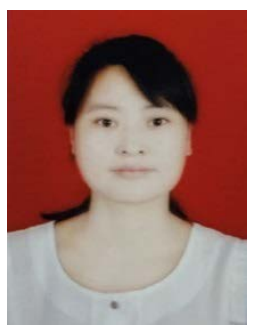

Xingjuan Cai received her Ph.D. degree in Control Science and Engineering from Tongji University, China, in 2017. She is an Associate Professor with the school of Computer Science and Technology, Taiyuan University of Science and Technology, Taiyuan, China. Her research interest includes bio-inspired computation and application.

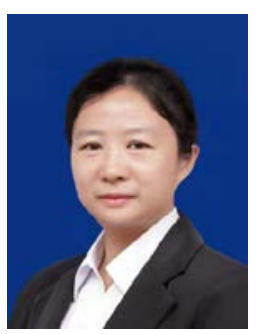

Liping Xie received the B.Sc. and M.Sc. degrees from Taiyuan University of Science and Technology, Taiyuan, China, in 2001 and 2005, respectively, and the Ph.D. degree from Lanzhou University of Technology, Lanzhou, China, in 2010. She is a Professor with the Department of Computer Science and Technology, Taiyuan University of Science and Technology. Her Current research interests include swarm intelligence, optimization algorithm, swarm robotics. 